This item is the archived peer-reviewed author-version of:

\title{
Plausible rice yield losses under future climate warming
}

\section{Reference:}

Zhao Chuang, Piao Shilong, Wang Xuhui, Huang Yao, Ciais Philippe, Elliott Joshua, Huang Mengtian, Janssens Ivan, Li Tao, Lian $\mathrm{Xu}, . .$. - Plausible rice yield losses under future climate warming

Nature plants - ISSN 2055-0278 - 3:1(2017), 16202

Full text (Publisher's DOI): http://dx.doi.org/doi:10.1038/NPLANTS.2016.202

To cite this reference: http://hdl.handle.net/10067/1404140151162165141 
2 Chuang Zhao ${ }^{1}$, Shilong Piao ${ }^{1,2,3}$, Xuhui Wang ${ }^{1}$, Yao Huang ${ }^{4}$, Philippe Ciais ${ }^{5}$, Joshua

3 Elliott $^{6}$, Mengtian Huang ${ }^{1}$, Ivan A Janssens ${ }^{7}, \mathrm{Tao} \mathrm{Li}^{8}, \mathrm{Xu} \mathrm{Lian}^{1}$, Yongwen Liu ${ }^{1}$,

4 Christoph Müller ${ }^{9}$, Shushi Peng ${ }^{1}$, Tao Wang ${ }^{2,3}$, Zhenzhong Zeng $^{1}$, Josep Peñuelas ${ }^{10,11}$

61 Sino-French Institute for Earth System Science, College of Urban and

7 Environmental Sciences, Peking University, Beijing 100871, China

$8{ }^{2}$ Key Laboratory of Alpine Ecology and Biodiversity, Institute of Tibetan Plateau

9 Research, Chinese Academy of Sciences, Beijing 100085, China

$10{ }^{3}$ Center for Excellence in Tibetan Earth Science, Chinese Academy of Sciences,

11 Beijing 100085, China

$12{ }^{4}$ State Key Laboratory of Vegetation and Environmental Change, Institute of Botany,

13 Chinese Academy of Sciences, Beijing 100093, China

$14{ }^{5}$ Laboratoire des Sciences du Climat et de l'Environnement, CEA CNRS UVSQ,

15 Gif-sur-Yvette, France

$16{ }^{6}$ University of Chicago Computation Institute, Chicago, IL 60637, United States of 17 America

$18{ }^{7}$ Department of Biology, University of Antwerp, Universiteitsplein 1, 2610 Wilrijk,

19 Belgium

$20 \quad{ }^{8}$ International Rice Research Institute, Los Baños, Philippines

$21{ }^{9}$ Potsdam Institute for Climate Impact Research, 14473 Potsdam, Germany

$22{ }^{10}$ CREAF, Cerdanyola del Valles, Barcelona 08193, Catalonia, Spain.

$23{ }^{11}$ CSIC, Global Ecology Unit CREAF-CEAB-CSIC-UAB, Cerdanyola del Valles,

24 Barcelona 08193, Catalonia, Spain. 
Rice is the staple food for more than $50 \%$ of the world's population ${ }^{1-3}$.

29 Reliable prediction of changes in rice yield is thus central for maintaining global

30 food security. This is an extraordinary challenge. Here, we compare the

31 sensitivity of rice yield to temperature increase derived from field warming

32 experiments and three modelling approaches: statistical models, local crop

33 models and global gridded crop models. Field warming experiments produce a

34 substantial rice yield loss under warming, with an average temperature

35 sensitivity of $-\mathbf{5 . 2} \pm 1.4 \% \mathrm{~K}^{-1}$. Local crop models give a similar sensitivity $(-6.3 \pm 0.4 \%$

$36 \mathbf{K}^{-1}$ ), but statistical and global gridded crop models both suggest less negative

37 impacts of warming on yields $\left(0.8 \pm 0.3 \% \mathrm{~K}^{-1}\right.$ and $-2.4 \pm 3.7 \% \mathrm{~K}^{-1}$, respectively).

38 Using data from field warming experiments, we further propose a conditional

39 probability approach to constrain the large range of global gridded crop model

40 results for the future yield changes in response to warming by the end of the

41 century (from $-1.3 \% \mathrm{~K}^{-1}$ to $-9.3 \% \mathrm{~K}^{-1}$ ). The constraint implies a more negative

42 response to warming $\left(-8.3 \pm 1.4 \% \mathrm{~K}^{-1}\right)$ and reduces the spread of the model

43 ensemble by 33\%. This yield reduction exceeds that estimated by the

44 International Food Policy Research Institute assessment $\left(-4.2 \text { to }-6.4 \% \mathrm{~K}^{-1}\right)^{4}$. Our

45 study suggests that without $\mathrm{CO}_{2}$ fertilization, effective adaptation and genetic

46 improvement, severe rice yield losses are plausible under intensive climate

47 warming scenarios. 
49 Hunger and malnutrition are two alarming problems calling for increased yields ${ }^{5,6}$.

50 Rice is currently one of the most widely grown crops in the world and the main source

51 of calories in developing countries ${ }^{1-3}$. Any reduction in rice productivity could,

52 therefore, have dramatic implications for global food security ${ }^{5}$. Climate warming

53 exceeding the optimum physiological temperature of rice plants has been shown to

54 cause such a reduction ${ }^{7,8}$. The assessment of food security from the International Food

55 Policy Research Institute (IFPRI) also stated that climate change, without the separate

56 effects of $\mathrm{CO}_{2}$ fertilization, would cause a $10-12 \%$ reduction of irrigated rice yield

57 globally by $2050^{4}$. Unfortunately, we have poor understanding of the physiological

58 mechanisms through which rice plants may respond to climate change. Many studies

59 are using process-based crop models to project climate change impacts on crop

60 yields $^{9-10}$. These models integrate plant-scale physiological mechanisms, and can be

61 run at site, regional or global scale with forcing variables derived from global climate

62 models under different greenhouse gas emission scenarios. Yet, the parameters of crop

63 models are usually not measured across the full scale of model applications, and

64 model equations may also be wrong, leading to large uncertainties in projections of

65 future climate change impacts ${ }^{10-12}$.

66

67 The Inter-Sectoral Impact Model Intercomparison Project (ISI-MIP-1) ${ }^{13}$ and the 
68 Agricultural Model Intercomparison and Improvement Project (AgMIP) ${ }^{14}$ coordinated

69 multi-model simulations of the yields of major crops, including rice. One of the

70 findings of AgMIP is that multi-model mean or median values give better simulations

71 of the observed yield of rice ${ }^{15}$ than any individual model, but it remains unclear

72 whether the 'average model' is meaningful at all. Errors in parameter values, as well

73 as in model structure, result in large model-to-model variation in simulated yield ${ }^{10}$.

74 However, if the bias of a model for the present persists into the future, an emerging

75 constraint can be established through which present-day observations can be used for

76 eliminating less realistic models in the simulation of temperature response; this

77 reduces the uncertainty in the ensemble projection. This heuristic approach called

78 'emerging constraint' has been applied to constrain simulations, e.g., of the sensitivity

79 of the tropical carbon cycle and of snow albedo, to temperature ${ }^{16,17}$. Here, to reduce

80 the large range of the ISI-MIP-1 global gridded crop models $\left(\right.$ GGCMs) ${ }^{18}$ for the

81 sensitivity of rice yield to temperature, we use a new compilation of data from 83

82 field warming experiments at 13 sites over the globe (Supplementary Table 1) (see

83 Methods).

84

85 Five GGCMs driven by daily weather outputs from five climate models (CM) (see

86 Methods) were run under the high warming Representative Concentration Pathway

87 RCP8.5 (2070-2099) scenario, with $\mathrm{CO}_{2}$ fixed at the present-day value (excluding the 
88 relevant benefits from $\mathrm{CO}_{2}$ fertilization in the future). This procedure allows us to

89 estimate the effect of climate change alone on yield. The five climate models used to

90 drive the GGCMs, gave an increase in growing-season mean air temperature over

91 rice-growing areas ranging from $3.3 \mathrm{~K}$ (GFDL-ESM2M) to 5.0 K (IPSL-CM5A-LR)

92 relative to today (Fig. 1a). The median value of the climate-induced rice yield change

93 was $-27 \%$ (Fig. 1b) - a large yield reduction which would pose a threat to future

94 food security. However, the range of model responses was large, reflecting

95 uncertainties in climate projections and in GGCMs, with yield reductions ranging

96 from 6.6\% in LPJ-GUESS+HadGEM2-ES to 42.4\% in EPIC+HadGEM2-ES (see also

97 ref.18). Dividing the changes in yield by the magnitude of temperature warming

98 above present-day values defines the long-term sensitivity of rice yield to warming by

99 the end of the twenty-first century $\left(S_{\mathrm{Y}, \mathrm{T}}^{\mathrm{lt}}\right)$. This sensitivity was negative for all

100 combinations of GGCM and climate model, and ranged from $-1.3 \% \mathrm{~K}^{-1}$ with

101 LPJ-GUESS+HadGEM2-ES to $-9.3 \% \mathrm{~K}^{-1}$ with EPIC+HadGEM2-ES; the median

102 value was $-6.5 \% \mathrm{~K}^{-1}$.

103

104 Then, for each GGCM-CM pair, we also calculated the present-day interannual

105 temperature sensitivity of rice yield $\left(S_{\mathrm{Y}, \mathrm{T}}^{\mathrm{int}}\right)$ for the model grid cells where the field

106 experiments were located, using multiple linear regression models to separate the

107 sensitivity of modelled yields (1971-2000) to growing-season temperature, 
108 precipitation and radiation. Figure $2 \mathrm{a}$ shows that there is an emerging strong linear 109 relationship $\left(R^{2}=0.75, \quad P<0.001\right)$ between long-term $\left(S_{\mathrm{Y}, \mathrm{T}}^{\mathrm{lt}}\right)$ and present-day 110 interannual $\left(S_{\mathrm{Y}, \mathrm{T}}^{\mathrm{int}}\right)$ sensitivities of yield to temperature across all GGCM-CM

111 combinations. This means that a model showing a high negative yield response to

112 warm years during the last 30 years also projects a high warming-induced yield

113 decrease in the future. This implies that the GGCM responses to temperature are

114 generally conserved between historical and future conditions.

116 To assess the realism of these modelled yield sensitivities to warming, we

117 compiled data from field experiments where rice plots were warmed (Supplementary

118 Table 1). More than $80 \%$ (67 out of 83) of the field experiments reported a rice yield

119 loss under warming, with an average observed sensitivity of yield to warming $\left(S_{\mathrm{Y}, \mathrm{T}}^{\mathrm{obs}}\right)$

120 of $-5.2 \pm 1.4 \% \mathrm{~K}^{-1}$ (Fig. 3). According to the 'emerging constraint' method (see

121 Methods), these field experiments provided an observation-based probability density

122 function (PDF) for modelled $S_{\mathrm{Y}, \mathrm{T}}^{\mathrm{int}}$, and the linear relationship between $S_{\mathrm{Y}, \mathrm{T}}^{\mathrm{lt}}$ and $S_{\mathrm{Y}, \mathrm{T}}^{\mathrm{int}}$

123 (Fig. 2a) provided another PDF of $S_{\mathrm{Y}, \mathrm{T}}^{\mathrm{lt}}$ for a given $S_{\mathrm{Y}, \mathrm{T}}^{\mathrm{int}}$. The conditional probability

124 of modelled $S_{\mathrm{Y}, \mathrm{T}}^{\mathrm{lt}}$ that is consistent with the PDF of observed sensitivities (red dashed

125 line in Fig. 2b) gives a PDF of constrained modelled $S_{\mathrm{Y}, \mathrm{T}}^{\mathrm{lt}}$. The maximum likelihood

126 value of this constrained $S_{\mathrm{Y}, \mathrm{T}}^{\mathrm{lt}}$ sensitivity was more negative $\left(-8.3 \pm 1.4 \% \mathrm{~K}^{-1}\right)$ than the

127 one of the original model ensemble (Fig. 2b), and the 1-sigma uncertainty of the PDF 
128 of $S_{\mathrm{Y}, \mathrm{T}}^{\mathrm{lt}}$ was reduced by $33 \%$. This means that the information from field warming

129 experiments shifts the modelled long-term temperature sensitivities of rice yield

130 towards more negative values, and reduces the variation among models. When

131 applying the same emerging constraint of the conditional probability to the model grid

132 cells of the experimental sites, or to the grid cells with similar climate or similar rice

133 yield, the constrained $S_{\mathrm{Y}, \mathrm{T}}^{\mathrm{lt}}$ values in all cases were more negative than the original

134 ensemble of models, and had a lower uncertainty (Supplementary Fig. 1).

136 The temperature sensitivities obtained from field experiments can also be

137 considered as realistic analogues of GGCM long-term sensitivities, because both

138 approaches consider a warming over ambient conditions of similar magnitude.

139 Replacing the present-day temperature sensitivities $\left(S_{\mathrm{Y}, \mathrm{T}}^{\mathrm{int}}\right)$ over the GGCM grid cells

140 of experimental sites (horizontal-axis variable) with that of the long-term ones $\left(S_{\mathrm{Y}, \mathrm{T}}^{\mathrm{lt}}\right)$

141 in Fig. 2, we found that the experimentally constrained $S_{\mathrm{Y}, \mathrm{T}}^{\mathrm{lt}}$ was $-7.2 \pm 1.5 \% \mathrm{~K}^{-1}$, still

142 less uncertain and more negative than the unconstrained value reflecting the spread of

143 all the GGCMs forced by different climate models (Supplementary Fig. 2).

145 With the emerging constraint approach of this study, it is important to assess all

146 the uncertainties that might bias the final result. For instance, some experiments

147 included multiple warming treatments and nutrient levels. We thus verified that $S_{\mathrm{Y}, \mathrm{T}}^{\mathrm{obs}}$ 
148 depends neither on the magnitude of warming applied (Supplementary Fig. 3, $P>0.1$ ),

149 nor on the background growing-season temperature (Supplementary Fig. 4, $P>0.1$ ) or

150 nutrient levels (Supplementary Fig. 5, $P>0.1$ ) across the set of experiments we have

151 compiled. In addition, field experiments had different designs and used different

152 techniques to warm the plots. Passive warming techniques using greenhouses or

153 open-top-chambers were criticized because they also alter light, wind, and soil

154 moisture ${ }^{19,20}$-active warming techniques using artificial heaters are considered more

155 reliable ${ }^{20,21}$. When only the results from active warming experiments were used

156 (Supplementary Fig. 6), the constrained $S_{\mathrm{Y}, \mathrm{T}}^{\mathrm{lt}}$ was $-7.0 \pm 1.7 \% \mathrm{~K}^{-1}$, remaining more

157 negative than the unconstrained value, but the uncertainty reduction achieved for

158 model results was smaller (only 19\% against 33\% with all experiments), which is

159 attributed mainly to the small number of active warming experiments published so far

160 (only five sites; Supplementary Table 1).

162 A second source of uncertainty in our approach is that the values of $S_{\mathrm{Y}, \mathrm{T}}^{\mathrm{lt}}$ derived

163 from model simulations represent the average yield change divided by the average

164 temperature increase averaged over many years with non-uniform warming across the

165 growing season, whereas field experiments last only a few years. Using individual

166 years, instead of the average of the last 30 years of the twenty-first century, to

167 calculate $S_{\mathrm{Y}, \mathrm{T}}^{\mathrm{lt}}$, the constrained $S_{\mathrm{Y}, \mathrm{T}}^{\mathrm{lt}}$ remained less uncertain and more negative than 
168 the unconstrained value for 29 individual years (Supplementary Fig. 7). Our result is

169 thus robust and not sensitive to the method used to define the long-term yield

170 sensitivity to warming in model outputs. In addition, warming experiments located in

171 the US (24 out of 83 experiments, Supplementary Table 1) might be not representative

172 of the varieties, edaphic and climate conditions over today's dominant rice growing

173 regions in Asia. However, even when using only the experiments performed on Asian

174 rice varieties, with only the GGCM grid cells of these regions, the emerging linear

175 relationship between $S_{\mathrm{Y}, \mathrm{T}}^{\mathrm{lt}}$ and $S_{\mathrm{Y}, \mathrm{T}}^{\mathrm{int}}$ was still present (Supplementary Fig. 8, $R^{2}=0.74$,

$176 P<0.001)$, and the constrained $S_{\mathrm{Y}, \mathrm{T}}^{\mathrm{lt}}$ was $-6.9 \pm 1.4 \% \mathrm{~K}^{-1}$, less uncertain than the

177 unconstrained value $\left(-5.8 \pm 2.0 \% \mathrm{~K}^{-1}\right)$.

178

179 Why does the ISI-MIP-1 ensemble median of pairs of GGCMs and climate

180 models underestimate rice yield losses in response to warming (Fig. 2b)? One reason

181 might be the inclusion of adaptation in some GGCMs. For instance, LPJ-GUESS

182 assumes very flexible adaptation in growing-season lengths, i.e., plasticity of cultivars,

183 and GEPIC allows for adaptation in sowing dates. Removing these two models from

184 the constraint, does not remove this underestimation (Supplementary Fig. 9),

185 suggesting that the fact that some models include a degree of adaptation does not

186 eliminate the underestimated $S_{\mathrm{Y}, \mathrm{T}}^{\mathrm{lt}}$ in GGCMs. Also, the use of CM-based climate

187 scenarios with non-uniform warming across the growing season and where also 
188 changes in radiation and precipitation are included, can lead to a veiled temperature 189 response. As most of the rice production is fully irrigated, we assume that the 190 temperature signal is the dominant climate impact also in the CM-driven GGCM 191 simulations. Another reason could be that the ensemble did not contain a sufficiently 192 large enough number of crop models (five in our study). All the possibilities of current 193 rice models may not have been included and this would hamper the strength of the 194 model ensemble ${ }^{10,15}$. Fortunately, a larger number of crop models will be used in the 195 Phase II of ISI-MIP/AgMIP; this will allow a further test of the robustness of the 196 emerging constraint approach.

198 Independently from field warming experiments and GGCMs, there are also a 199 large number of publications from local crop models used to interpret field trials 200 (arguably those models are well calibrated to specific rice varieties and cultivation 201 practice) and from statistical models where the sensitivity of rice yield to temperature 202 change is derived from observed interannual variability. These different temperature 203 sensitivities are shown in Fig. 3 for the present-day period and the future (end of the 204 century). For the present-day sensitivities, 95\% of local crop model simulations (329 205 studies out of 346) give a negative response to warming, with a mean sensitivity of $206-6.3 \pm 0.4 \% \mathrm{~K}^{-1}$, more negative but consistent with the values inferred from field 207 warming experiments $\left(-5.2 \pm 1.4 \% \mathrm{~K}^{-1}\right)$. Statistical models have a surprisingly lower 
208 percentage of studies (46 studies out of 77) presenting negative $S_{\mathrm{Y}, \mathrm{T}}$ than warming

209 experiments (more than $80 \%$ of studies), and also give a weaker mean sensitivity

$210\left(S_{\mathrm{Y}, \mathrm{T}}=-0.8 \pm 0.3 \% \mathrm{~K}^{-1} ;\right.$ Fig. 3$)$ than both warming experiments and local crop models.

211 This weak sensitivity might be due to the aggregated nature and disputable quality of

212 historical yield and weather data in different regions ${ }^{22}$, to difficulties in separating the

213 temperature effect from co-varying management practice ${ }^{23}$, increasing $\mathrm{CO}_{2}$, and to

214 non-linearity in the temperature response ${ }^{24}$. Lower sensitivities are also found in the

215 GGCM results during the present-day period compared to the long term (Fig. 3). This

216 suggests that GGCMs have thresholds above which the temperature response of rice

217 yield becomes significantly more negative (see also ref. 18).

219 We also compared our $S_{\mathrm{Y}, \mathrm{T}}^{\mathrm{lt}}$ value with that implied from IFPRI (as a 220 representative of the policy community) who project the future of the world's food 221 supply. They predicted 10 and $12 \%$ losses of global rice yield by 2050 , based on

222 temperature increase scenarios of $1.5{ }^{\circ} \mathrm{C}$ and $2.9{ }^{\circ} \mathrm{C}$, respectively ${ }^{4}$. Thus a rough

223 estimate of the sensitivity of rice yield to warming is -4.2 to $-6.4 \% \mathrm{~K}^{-1}$, a smaller

224 magnitude than that from the global crop models constrained by experimental data in

225 our study $\left(-8.3 \pm 1.4 \% \mathrm{~K}^{-1}\right)$. However, we noted that the constrained $S_{\mathrm{Y}, \mathrm{T}}^{\mathrm{lt}}$ derived here

226 was for the end of this century (2070-2099), inconsistent with the time frame used by

227 IFPRI (2050s). When applying the emerging constraint to the time frame of 
228 mid-century (2036-2065), the constrained $S_{\mathrm{Y}, \mathrm{T}}^{\mathrm{lt}}$ was $-8.5 \pm 2.3 \% \mathrm{~K}^{-1}$ (Supplementary

229 Fig. 10) - still a larger magnitude than the number from IFPRI. This result suggests

230 that warming appears to present an even greater challenge to rice than expected and

231 more effective adaptation strategies are thus required.

233 The prediction of yield loss under future warming notably does not consider

234 other-than-climate factors that could sustain or increase yield, in particular increased

$235 \mathrm{CO}_{2}^{25,26}$, adaptation ${ }^{11,27}$ and improved management/cultivars that are independent of

236 adaptation to warmer temperatures ${ }^{28}$. For instance, the current rates of genetic gains in

237 yield for hybrid rice are $0.6-0.7 \% \mathrm{yr}^{-1}$ (ref. 28). In our study, the results from the

238 global gridded crop model constrained by observations suggest a yield loss of $37 \%$ for

239 the end of the century due to increased temperature under the RCP8.5 scenario

240 (multiply the constrained sensitivity in Fig. 2 by climate warming in Fig. 1), but the

241 loss will unfold over 70 years, i.e., at an average rate of $0.5 \% \mathrm{yr}^{-1}$. The genetic

242 improvement sustained during one century at current rates could thus offset the

243 negative impact from increased temperature. To fulfil the projected increase in cereal

244 demand for the world population $\left(\sim 1.2 \% \mathrm{yr}^{-1}\right)^{29}$, however, the increase in rice yield

245 from technological change, together with the $\mathrm{CO}_{2}$ effect and adaptation, would need

246 to be much higher $\left(1.7 \% \mathrm{yr}^{-1}\right)$ to offset the development of negative effects of climate

247 change at a rate of $0.5 \% \mathrm{yr}^{-1}$. 
249 Our study, combining field warming experiments with three modelling

250 approaches, comprehensively assessed the global response of rice yield to warming.

251 The main result is that all approaches indicated a decrease in rice yield in response to

252 warming, and the field warming experiments suggested an even higher risk of future

253 yield reductions than that inferred from unconstrained GGCM results. Future

254 experiments with standard measurement protocols, long time periods and a large

255 range of rice genotypes and management types ${ }^{30}$ should provide more insight on

256 constraining modelling results. Our results, however, show that warming under

257 climate change poses a significant threat to rice production and thus to a major staple

258 food with substantial impact on the food security of developing and emerging

259 economies. The long-term perspective of climate change allows us to prepare

260 agricultural production systems for this challenge, but suitable policies must be put in

261 place in the near future, given that targeted research on adaptation options and their

262 large-scale implementation will require considerable time.

264 Methods

265 ISI-MIP data set. Starting in 2012, the Inter-Sectoral Impact Model Intercomparison

266 Project (ISI-MIP-Phase 1 project; isi-mip.org) used multi-model ensembles to assess

267 the climate change impacts across multiple sectors. In the agriculture sector, multiple 
268 global gridded crop models $(\mathrm{GGCMs})^{18}$ were used to simulate crop yield. We used

269 yield simulated by five GGCMs (EPIC, GEPIC, LPJ-GUESS, LPJmL and pDSSAT).

270 These model outputs are available as annual time series at a spatial resolution of $0.5 \times$

2710.5 degrees. GGCM simulations were driven by historical (1971-2005) and future

272 (2006-2099) climate forcing including temperature, precipitation and solar radiation.

273 These forcing data were taken from a bias-corrected climate data set based on five

274 climate models (CMs) in the Coupled Model Intercomparison Project Phase 5

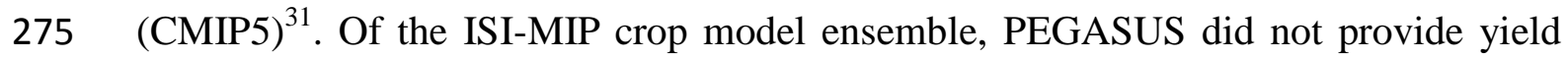

276 data for rice and GAEZ-IMAGE was excluded because its modelling approach does

277 not provide sufficient information on interannual variability to calculate the

278 temperature sensitivity of rice yield. More detailed information about the five

279 GGCMs which were used can be found in ref.18. The high-emission scenario,

280 representative concentration pathway (RCP) 8.5 was chosen as it not only represents

281 the upper end of projected climate change, but also provides the largest ensemble of

282 GGCM-CM combinations to consider the broadest possible range of climate impacts.

283 GEPIC and LPJ-GUESS only contributed data for one CM (i.e., HadGEM2-ES) and

284 thus a total of seventeen GGCM-CM combinations were used in our analysis. All

285 GGCM-CM simulations used here were conducted with constant $\mathrm{CO}_{2}$ concentration

286 and current management (see ref. 18 for exceptions). We used the model output for

287 the full irrigation scenario, since irrigated rice currently makes up about $75 \%$ of world 
288 production $^{3}$.

290 Literature review. We searched peer-reviewed and primary research from Web of

291 Science, Google Scholar and China National Knowledge Infrastructure (CNKI,

292 http://www.cnki.net) that was published before January 2015. All publications related

293 to the responses of rice yield to temperature change were considered. Three main

294 approaches were distinguished, namely, local process-based crop models, statistical

295 models and field warming experiments. To obtain the sensitivity of rice yield to

296 temperature $\left(S_{\mathrm{Y}, \mathrm{T}}\right.$; yield change per K), local process-based models usually conduct an

297 arbitrary sensitivity test (e.g., $+2{ }^{\circ} \mathrm{C}$ scenario), with other conditions kept constant;

298 whereas statistical models use regression equations to relate historical records of rice

299 yield to weather including temperature. On the other hand, field warming experiments

300 apply direct warming treatments to rice in field plots. $S_{\mathrm{Y}, \mathrm{T}}$ is calculated as :

$$
S_{\mathrm{Y}, \mathrm{T}}=\Delta Y / \Delta T
$$

302 where $\Delta Y$ and $\Delta T$ are the rice yield change and temperature change, respectively. The

303 average $S_{\mathrm{Y}, \mathrm{T}}$ and its uncertainty for experiments are obtained from bootstrap

304 resmpling. Here, we denote the experimental data (Supplementary Table 1) as: $X=\left\{X_{1}\right.$, $\left.305 X_{2}, \ldots, X_{n}\right\}$, where $X_{n}$ represent all the experiments at site $n$. The steps of 306 bootstrapping are as follows: (1), randomly resample one experiment at each site to 307 obtain a bootstrap resample: $X_{1} *=\left(x_{1}, x_{2}, \ldots, x_{n}\right)$, where $x_{n}$ represents the sampled 
308 experiment at site $n$. (2), compute the mean of this resample and obtain the first

309 bootstrap mean: $\mu_{1}^{*}=\frac{1}{n} \sum_{1}^{\mathrm{n}} x_{\mathrm{i}}$. (3), repeat the processes of (1) and (2) to obtain the

310 second resample $X_{2}^{*}$ and compute the second bootstrap mean $\mu_{2} *$. Repeating this

3115000 times, we have $\mu_{1}^{*}, \mu_{2} *, \ldots, \mu_{5000} *$, which constitute an empirical bootstrap

312 distribution (PDF) of the sample mean. Here, each $\mu^{*}$ represents one case of average

313 temperature sensitivity across all the sites (Figure R1 and Supplementary Fig. 11).

314 The difference among the $5000 \mu^{*}$ values originates from the use of different

315 experiments within the sites in each resampling. Therefore, the PDF now reflects the

316 variations caused by different experiments within sites. To ensure comparability, we

317 then estimated the uncertainties of temperature sensitivity for local crop models and

318 statistical models in the same way.

320 Constraint. Our constraint methodology comes from Cox et al. ${ }^{16}$, who built an

321 emergent linear relationship between the sensitivity of tropical land-carbon storage to

322 warming and the sensitivity of the annual growth rate of atmospheric $\mathrm{CO}_{2}$ to tropical

323 temperature anomalies across models. They then used the historical observed $\mathrm{CO}_{2}$

324 growth rate sensitivity to temperature to constrain the uncertainties of future climate

325 impact on tropical carbon through the conditional probability approach. Here we used

326 a similar approach, first building the relationship between the historical temperature

327 sensitivity of crop yield and the future yield feedbacks across the GGCM 
328 model-ensembles, and then using the observed field warming experiments to constrain

329 future modelled yield-climate feedbacks. The details of the constraint methods are

330 described in Supplementary Methods. It should be noted that the PDF of GGCM-CM

331 could be biased, because some crop models (GEPIC and LPJ-GUESS) were only

332 paired with one CM (HadGEM2-ES). This unbalance in the selection of the

333 GGCM-CM combination was checked with five GGCMs but with random selection

334 of different CMs, i.e., one pair of GGCM-CMs with random CM selection

335 (Supplementary Fig. 12).

336

337 Data availability. The data supporting the findings of our study are accessible within

338 the article and Supplementary Information files.

340 References

341 1. FAOSTAT, http://faostat.fao.org/default.aspx (2014).

342 2. Seck, P. A., Diagne, A., Mohanty, S. \& Wopereis M. C. Crops that feed the world

343 7: rice. Food Secur. 4, 7-24 (2012).

344 3. Maclean, J. L., Dawe, D. C., Hardy, B. \& Hettel, G. P. Rice Almanac: Source

345 Book for the Most Important Economic Activity on Earth 3rd edn (CABI

$346 \quad$ Publishing, 2002).

347 4. Nelson, G. C. et al. Food security, farming, and climate change to 2050: 
348 Scenarios, results, policy options (IFPRI, Washington DC, 2010).

349 5. Godfray, H. C. et al. Food security: The challenge of feeding 9 billion people. $350 \quad$ Science 327, 812-818 (2010).

351 6. Foley, J. A. et al. Solutions for a cultivated planet. Nature 478, 337-342 (2011).

352 7. Gourdji, S. M., Sibley, A. M. \& Lobell, D. B. Global crop exposure to critical high 353 temperatures in the reproductive period: historical trends and future 354 projections. Environ. Res. Lett. 8, 024041 (2013).

355 8. Sánchez, B., Rasmussen, A. \& Porter, J. R. Temperatures and the growth and 356 development of maize and rice: a review. Global Change Biol. 20, 408-417 $357 \quad$ (2014).

358 9. Rosenzweig, C. \& Parry, M. L. Potential impact of climate change on world food $359 \quad$ supply. Nature 367, 133-138 (1994).

360 10. Asseng, S. et al. Uncertainty in simulating wheat yield under climate change. $361 \quad$ Nature Clim. Change 3, 827-832 (2013).

362 11. Challinor, A. J. et al. A meta-analysis of crop yield under climate change and 363 adaptation. Nature Clim. Change 4, 287-291 (2014).

364 12. Rötter, R. P., Carter, T. R., \& Olesen, J. E. Crop-climate models need an 365 overhaul. Nature Clim. Change 1, 175-177 (2011).

366 13. Warszawski, L. et al. The Inter-Sectoral Impact Model Intercomparison Project 367 (ISI-MIP): Project framework. Proc. Natl. Acad. Sci. USA 111, 3228-3232 

(2014).

369 14. Rosenzweig, C. et al. The agricultural model intercomparison and improvement 370 project (AgMIP): protocols and pilot studies. Agric. For. Meteorol. 170, 166-182 $371 \quad$ (2013).

372 15. Li, T. et al. Uncertainties in predicting rice yield by current crop models under a 373 wide range of climatic conditions. Global Change Biol. 21, 1328-1341 (2015).

374 16. Cox, P. M. et al. Sensitivity of tropical carbon to climate change constrained by 375 carbon dioxide variability. Nature 494, 341-344 (2013).

376 17. Hall, A. \& Qu, X. Using the current seasonal cycle to constrain snow albedo 377 feedback in future climate change. Geophys. Res. Lett. 33, L03502, 378 doi:10.1029/2005GL025127 (2006).

379 18. Rosenzweig, C. et al. Assessing agricultural risks of climate change in the $21 \mathrm{st}$ 380 century in a global gridded crop model intercomparison. Proc. Natl. Acad. Sci. $381 \quad$ USA 111, 3268-3273 (2014).

382 19. Kimball, B. A. Theory and performance of an infrared heater for ecosystem 383 warming. Global Change Biol. 11, 2041-2056 (2005).

384 20. Aronson, E. L. \& McNulty, S. G. Appropriate experimental ecosystem warming 385 methods by ecosystem, objective, and practicality. Agric. For. Meteorol. 149, $386 \quad 1791-1799(2009)$.

387 21. De Boeck, H. J. \& Nijs, I. An alternative approach for infrared heater control in 
warming and extreme event experiments in terrestrial ecosystems. J. Ecol.99, 724-728 (2011).

22. Lobell, D. B. et al. Prioritizing climate change adaptation needs for food security in 2030. Science 319, 607-610 (2008).

23. Maltais-Landry, G. \& Lobell, D. B. Evaluating the contribution of weather to maize and wheat yield trends in 12 U.S. counties. Agron. J. 104, 301-311 (2012).

394 24. Schlenker, W. \& Roberts, M. J. Nonlinear temperature effects indicate severe 395 damages to US crop yield under climate change. Proc. Natl. Acad. Sci. USA 106, 15594-15598 (2009).

397 25. Ziska, L. H. \& Bunce, J. A. Predicting the impact of changing $\mathrm{CO}_{2}$ on crop yields: 398 some thoughts on food. New Phytol. 175, 607-618 (2007).

399 26. Ainsworth, E. A. Rice production in a changing climate: a meta-analysis of 400 responses to elevated carbon dioxide and elevated ozone concentration. Global $401 \quad$ Change Biol. 14, 1642-1650 (2008).

402 27. Howden, S. M. et al. Adapting agriculture to climate change. Proc. Natl. Acad. Sci. USA 104, 19691-19696 (2007).

404 28. Hall, A. J. \& Richards, R. A. Prognosis for genetic improvement of yield potential 405 and water-limited yield of major grain crops. Field Crop. Res. 143, 18-33 (2013).

406 29. Fisher, G. How do climate change and bioenergy alter the long-term outlook for 407 food, agriculture and resource availability? (FAO, Rome, 2009). 
408 30. Porter, J. R. et al. in Climate Change 2014: Impacts, Adaptation and 409 Vulnerability: Contribution of Working Group II to the Fifth Assessment Report

410 of the Intergovernmental Panel on Climate Change, Field, C. B. et al., Eds.

411 (Cambridge Univ. Press, Cambridge, 2014).

412 31. Hempel, S., Frieler, K., Warszawski, L., Schewe, J. \& Piontek, F. A 413 trend-preserving bias correction-the ISI-MIP approach. Earth Syst. Dyn. 4, $414 \quad 219-236(2013)$.

\section{Acknowledgements}

417 We thank the Intersectoral Impact Model Intercomparison Project (ISI-MIP) and 418 the Agricultural Model Intercomparison and Improvement Project (AgMIP) for 419 providing crop model simulation results. We also thank Dr. Senthold Asseng for 420 helpful comments. This study was supported by the National Natural Science 421 Foundation of China (41530528 and41561134016), 111 project (B14001), and 422 National Youth Top-notch Talent Support Program in China. P.C., I.J. and J.P. research 423 was supported by the European Research Council Synergy grant 424 ERC-2013-SYG-610028, IMBALANCE-P. C.M. acknowledges financial support 425 from the MACMIT project (01LN1317A) funded through the German Federal 426 Ministry of Education and Research (BMBF).

\section{Author contributions}


428 S.L.P. designed research; C.Z. performed analysis; and all authors contributed to 429 the interpretation of the results and the writing of the paper.

\section{$430 \quad$ Author information}

431 Reprints and permissions information is available at www.nature.com/reprints.

432 The authors have no competing financial interests. Correspondence and requests for 433 materials should be addressed to S.L.P. (slpiao@ pku.edu.cn)

434 
436 Figure 1. Future climate change (2070-2099, RCP 8.5) and its impact on global

437 rice yield (in comparison to 1971-2000 baseline) from an ensemble of seventeen

438 GGCM-CMs without $\mathrm{CO}_{2}$ fertilization effects. a, Growing-season temperature

439 change $(\Delta T)$. b, Relative yield change ( $\Delta$ Yield). c, The long-term sensitivity of rice

440 yield to climate change $\left(S_{\mathrm{Y}, \mathrm{T}}^{\mathrm{lt}}\right)$. The dashed lines represent the median value of the

441 ensemble. GFDL, HadGEM2, IPSL, MIROC, and NorESM1 represent the climate

442 models GFDL-ESM2M, HadGEM2-ES, IPSL-CM5A-LR, MIROC-ESM-CHEM and

443 NorESM1-M, respectively.

444

445 Figure 2. Constraint on the long-term sensitivity of rice yield to temperature

446 change. a, The relationship between global long-term temperature sensitivity of rice

447 yield $\left(S_{\mathrm{Y}, \mathrm{T}}^{\mathrm{lt}}\right)$ and site-scale present-day rice yield sensitivity to temperature across an

448 ensemble of seventeen GGCM-CMs. The red line shows the temperature sensitivity

449 estimates $\left(S_{\mathrm{Y}, \mathrm{T}}^{\mathrm{obs}}\right.$, mean \pm standard deviation) from field warming experiments. b,

450 Probability distribution of $S_{\mathrm{Y}, \mathrm{T}}^{\mathrm{lt}}$. The black line in $\mathbf{b}$ is the probability distribution of

451 unconstrained $S_{\mathrm{Y}, \mathrm{T}}^{\mathrm{lt}}$, assuming all the components of the ensemble can be represented

452 by a Gaussian distribution; the red dashed line is the experimental data-constrained

453 probability distribution of $S_{\mathrm{Y}, \mathrm{T}}^{\mathrm{lt}}$.

454 
455 Figure 3. The estimates of sensitivity of rice yield to temperature change from

456 four distinct approaches. a, Map of the study sites from local crop models, statistical

457 models and field warming experiments. The regional-scale studies are represented by

458 the corresponding label in the centre of the region (one global-scale study is not

459 shown). Map was created using Matlab R2014b. b. The estimates of all the

460 present-day and long-term sensitivity of rice yield to temperature change $\left(S_{\mathrm{Y}, \mathrm{T}}\right)$. The

$461 S_{\mathrm{Y}, \mathrm{T}}$ from GGCMs are averages of all the global grid cells but not the grid cells where

462 field warming experiments are located. Error bars show the standard deviation. 